\title{
Ursache von Muskelschwäche: Spektrum neuromuskulärer Erkrankungen und diagnostisches Vorgehen
}

\section{Cause of Muscle Weakness: Spectrum of Neuromuscular Disorders and Diagnostic Approach}

Autoren

Maren Fitzner ${ }^{1}$, Jens Schmidt ${ }^{1,2,3}$

Institute

1 Klinik für Neurologie, Universitätsmedizin Göttingen, Göttingen, Deutschland

2 Abteilung Neurologie und Schmerztherapie, Immanuel Klinik Rüdersdorf, Universitätsklinikum der Medizinischen Hochschule Brandenburg, Rüdersdorf bei Berlin, Deutschland

3 Fakultät für Gesundheitswissenschaften Brandenburg, Medizinische Hochschule Brandenburg Theodor Fontane, Rüdersdorf bei Berlin, Deutschland

\section{Schlüsselwörter}

Muskelschwäche, Neuromuskuläre Erkrankung, Myopathie

Key words

muscle weakness, myopathy, neuromuscular disorder

Bibliografie

Akt Rheumatol 2021; 46: 373-381

DOI 10.1055/a-1548-8821

ISSN 0341-051X

(c) 2021. Thieme. All rights reserved.

Georg Thieme Verlag KG, Rüdigerstraße 14,

70469 Stuttgart, Germany

Korrespondenzadresse

Jens Schmidt

Abteilung Neurologie und Schmerztherapie

Immanuel Klinik Rüdersdorf

Universitätsklinikum der Medizinischen Hochschule

Brandenburg

Seebad 82/83

15562 Rüdersdorf bei Berlin

Deutschland

Tel. : + 4933638 83347, Fax: +4933638 83311

jens.schmidt@mhb-fontane.de

\section{ZUSAMMENFASSUNG}

Das Leitsymptom der muskulären Schwäche kann auf das Vorliegen einer neuromuskulären Erkrankung zurückzuführen sein, findet sich jedoch auch bei einer Reihe anderer Faktoren wie Elektrolytveränderungen, Vitaminmangel, Schilddrüsenerkrankungen, Bewegungsmangel oder bei unerwünschten Arzneimittelnebenwirkungen. Einer gründlichen Anamnese und körperlichen Untersuchung kommt daher in der differentialdiagnostischen Abklärung eine große Bedeutung zu. Die zeitliche Entwicklung, das Verteilungsmuster sowie Begleitsymptome erlauben oftmals bereits eine erste diagnostische Einordnung. Bei begründetem Verdacht auf das Vorliegen einer neuromuskulären Erkrankung sollte die weiterführende Diagnostik durch einen Neurologen oder ein neuromuskuläres Zentrum erfolgen. Zu den wichtigsten neuromuskulären Erkrankungen mit Leitsymptom einer muskulären Schwäche gehören die neuromuskulären Übertragungsstörungen, Neuropathien, erbliche und erworbene Myopathien sowie Motoneuronerkrankungen. Diese Übersicht stellt die wichtigsten Ursachen für eine muskuläre Schwäche zusammen und zeigt das zu empfehlende diagnostische Vorgehen auf.

\section{ABSTRACT}

Muscle weakness as a lead symptom can be caused by a neuromuscular disorder, but is also seen in non-muscular conditions such as electrolyte imbalance, vitamin deficiency, thyroid disorders, lack of exercise, unwanted drug effects etc. For quick and reliable workup, a precise history and clinical examination may be the key to diagnosis. Symptom development over time, pattern of weakness and concomitant conditions often allow for an initial differential diagnosis. In case of a suspected neuromuscular disorder, thorough diagnostic workup should be provided by a neurologist with neuromuscular expertise or by a neuromuscular centre. The most important neuromuscular conditions leading to weakness include neuromuscular transmission disorders such as myasthenia gravis, neuropathies, acquired and hereditary myopathies and motor neuron disorders. This review summarises the most important causes of muscle weakness and presents the suggested diagnostic approach. 


\section{Einleitung}

Die Ursachen für eine muskuläre Schwäche sind vielfältig: Nährstoffmangel, fehlendes Training oder zunehmendes Alter können der Grund für eine muskuläre Schwäche sein, aber auch Nebenwirkungen verschiedener Medikamente. Nicht selten weist eine muskuläre Schwäche als Leitsymptom jedoch auf das Vorliegen einer neuromuskulären Erkrankung hin. Zur Gruppe der neuromuskulären Erkrankungen gehören bspw. Neuropathien, neuromuskuläre Übertragungsstörungen wie die Myasthenia gravis, sowie erbliche und erworbene Muskelerkrankungen wie die idiopathischen inflammatorischen Myopathien. Aufgrund der Heterogenität der Erkrankungen sowie ihrer Seltenheit, ist die Diagnosestellung nicht selten erschwert und verzögert. Die Abklärung des Symptoms der muskulären Schwäche sollte daher gründlich durch Erhebung einer detaillierten Anamnese und Medikamentenanamnese, einer eingehenden körperlichen und klinisch-neurologischen Untersuchung und entsprechenden Zusatzdiagnostik erfolgen. Sollte sich der Verdacht auf das Vorliegen einer neuromuskulären Erkrankung erhärten, empfiehlt sich die Überweisung an einen Neurologen oder ein neuromuskuläres Zentrum.

\section{Symptom der Muskelschwäche}

Eine Muskelschwäche macht sich in der Regel durch eine fehlende Kraftentfaltung und/oder vorzeitigen Ermüdbarkeit der Muskulatur bemerkbar. Manchmal finden sich jedoch auch Symptome wie bspw. eine zunehmende Gangstörung, die nicht primär auf ein muskuläres Symptom schließen lassen. Die Muskelschwäche kann umschrieben oder generalisiert auftreten und dabei permanent, intermittierend oder klar belastungsabhängig bestehen. Häufig liegen weitere Begleitsymptome wie beispielsweise Myalgien, Muskelatrophien oder ein veränderter Muskeltonus vor. Aus zeitlichem Verlauf und Symptomkonstellation sowie eventuellen Begleitsymptomen lässt sich in der Regel bereits eine erste Eingrenzung der möglichen Differenzialdiagnosen vornehmen.

Zunächst sollte daher eine detaillierte Anamnese über die zeitliche Entwicklung der Symptome, das Verteilungsmuster der Muskelschwäche und die damit verbundenen Einschränkungen im Alltag, sowie die Abfrage relevanter Begleitsymptome bzw. Begleiterkrankungen erfolgen. Darüber hinaus sollte nach neuromuskulären Erkrankungen im familiären Umfeld gefragt und eine detaillierte Medikamentenanamnese erhoben werden.

Eine langsam progrediente Muskelschwäche findet sich bspw. bei erblichen Myopathien, Motoneuronerkrankungen oder langsam verlaufenden Neuropathien. Eine subakute Entwicklung der muskulären Schwäche lässt hingegen eher an das Vorliegen einer entzündlichen Myopathie, eine neuromuskuläre Übertragungsstörung, ein Guillain-Barré-Syndrom (GBS) oder eine medikamentöstoxische Genese denken. Apoplektiform aufgetretene Paresen müssen immer an eine zentrale Ursache wie eine zerebrale Ischämie oder Blutung denken lassen.

Verschiedene lonenkanalerkrankungen, endokrine oder metabolische Myopathien können zu episodischen Muskelparesen führen. Eine rein belastungsabhängige Muskelschwäche ohne Schmerzen lenkt den Verdacht in erster Linie auf das Vorliegen einer neuromuskulären Übertragungsstörung.
Das Verteilungsmuster der Muskelschwäche kann ebenfalls Aufschluss über die Ursache geben. Ein symmetrischer und proximaler Befall der Muskulatur findet sich bei den entzündlichen Myopathien, den meisten erblichen Myopathien wie bspw. der Muskeldystrophie Typ Duchenne und Typ Becker, sowie den metabolischen Myopathien. Seltene distale Myopathien sowie die meisten Polyneuropathien führen hingegen zu einer distal betonten, symmetrischen Muskelschwäche und -atrophie. Es finden sich jedoch auch Unterformen mit asymmetrischer oder fokaler Muskelschwäche wie zum Beispiel im Rahmen einer diabetischen Amyotrophie, der multifokal motorischen Neuropathie (MMN) oder zu Erkrankungsbeginn einer Amyotrophen Lateralsklerose (ALS). Eine Schwäche der Augenmuskeln findet sich bei den myasthenen Syndromen und äußert sich im Sinne einer Ptosis und Diplopie.

Zudem kann es im Rahmen multipler neuromuskulärer Erkrankungen zu einer Beteiligung der Schlund- oder Atemhilfsmuskulatur kommen. Diese findet sich am häufigsten bei der ALS und Myasthenia gravis (MG). Auch im Zuge von Myopathien wie beispielsweise der Einschlusskörpermyositis kann es zu einer Schluckstörung kommen. Der Beachtung dieses Symptoms oder aber auch die Suche nach einer subklinischen Schluckstörung kommt große Bedeutung bei aufgrund der Gefahr einer Aspirationspneumonie.

\section{Begleitsymptome}

Neben der muskulären Schwäche können je nach Ursache weitere muskuläre und nicht-muskuläre Symptome vorliegen. Patienten mit einer entzündlichen oder metabolischen Myopathie berichten häufig von Myalgien. Hier gilt es auch nach einer möglichen Abhängigkeit der Schmerzen von muskulärer Belastung bzw. einer Besserung unter Belastung zu fragen. Patienten mit einer Glykogenose Typ V (Mc Ardle) berichten über eine mögliche Besserung der Symptomatik im weiteren Verlauf der muskulären Belastung oder nach einer kurzen Ruhepause. Auch beim Lambert-Eaton-Myasthenie-Syndrom kann es nach kurzer starker Belastung zu einer vorübergehenden Besserung der Muskelkraft kommen. Muskelkrämpfe sind ein weiteres häufiges Symptom metabolischer Myopathien.

Nicht selten berichten Patienten selbst gar nicht das eigentliche Symptom einer muskulären Schwäche, sondern beschreiben eine zunehmende Gangstörung und/oder Sturzneigung sowie Einschränkungen der Griffkraft oder Feinmotorik. Bei der Myasthenia gravis kommt es zu Beginn oft zu isoliert auftretenden Doppelbildern als Ausdruck einer Beteiligung der äußeren Augenmuskeln.

Sensible Begleitsymptome wie Taubheitsgefühle, Kribbelparästhesien, Störungen der Tiefensensibilität oder neuropathische Schmerzen weisen hingegen auf eine neuropathische Ursache der Beschwerden, z. B. im Rahmen einer Polyneuropathie, hin. Häufigstes alltagsrelevantes Symptom einer im Vordergrund stehenden Einschränkung der Tiefensensibilität ist wiederum eine zunehmende Gangunsicherheit.

Die Gruppe der idiopathischen inflammatorischen Myopathien müssen als systemische Erkrankung verstanden werden. Es kann zu einer extramuskulären Organbeteiligung kommen. Somit muss nach weiteren Symptomen wie Hautveränderungen, einer Lungenbeteiligung, kardialen Symptomen und Malignomen gesucht werden. Auch eine Überlappung mit anderen Kollagenosen im Rahmen sogenannter Overlap-Syndrome sind möglich, so dass weitere Sym- 
ptome wie Fieber, Raynaud-Symptomatik, Sicca-Syndrom oder Arthralgien vorliegen können. Das Lambert-Eaton-Myasthenie-Syndrom sowie eine Polyneuropathie können als paraneoplastisches Syndrom auftreten. Zudem ist für die Dermatomyositis, Polymyositis und nekrotisierende Myopathie eine Assoziation mit Tumorerkrankungen bekannt.

Eine sorgfältige Anamnese auch im Hinblick auf eine mögliche B-Symtpomatik und eingehende körperliche Untersuchung sind somit unerlässlich.

- Tab. 1 gibt eine Übersicht über weitere klinische Aspekte und mögliche erste differentialdiagnostische Überlegungen bei Vorliegen einer Muskelschwäche.

\ Tab. 1 Klinische Aspekte bei Muskelschwäche

\begin{tabular}{|c|c|}
\hline & Mögliche Ursachen \\
\hline \multicolumn{2}{|l|}{ Zeitlicher Verlauf } \\
\hline " akut & Zerebrale Ischämie, intrazerebrale Blutung \\
\hline $\begin{array}{l}\text { " subakut/progre- } \\
\text { dient }\end{array}$ & $\begin{array}{l}\text { Myositis, neuromuskuläre Übertragungsstörun- } \\
\text { gen wie Myasthenia gravis, GBS, Multiple } \\
\text { Sklerose }\end{array}$ \\
\hline $\begin{array}{l}\text { - langsam progre- } \\
\text { dient }\end{array}$ & $\begin{array}{l}\text { Erbliche Myopathien, Motoneuronerkrankun- } \\
\text { gen wie Amyotrophe Lateralsklerose (ALS), } \\
\text { Polyneuropathie }\end{array}$ \\
\hline $\begin{array}{l}\text { - belastungsabhängig } \\
\text { oder episodisch }\end{array}$ & $\begin{array}{l}\text { Metabolische Myopathie, neuromuskuläre } \\
\text { Übertragungsstörung wie Myasthenia gravis } \\
\text { (MG) }\end{array}$ \\
\hline \multicolumn{2}{|l|}{ Art der Parese } \\
\hline - zentral/spastisch & $\begin{array}{l}\text { ALS, Schlaganfall, zerebrale Raumforderung, } \\
\text { Multiple Sklerose }\end{array}$ \\
\hline " peripher/schlaff & $\begin{array}{l}\text { Polyneuropathie, Vorderhornerkrankung, } \\
\text { Myopathie }\end{array}$ \\
\hline \multicolumn{2}{|l|}{ Verteilungsmuster } \\
\hline $\begin{array}{l}\text { " proximal symmet- } \\
\text { risch }\end{array}$ & $\begin{array}{l}\text { Erbliche oder entzündliche Myopathien, } \\
\text { neuromuskuläre Übertragungsstörungen wie } \\
\text { das Lambert-Eaton-Mastheniesyndrom (LEMS) } \\
\text { bzw. MG }\end{array}$ \\
\hline " distal symmetrisch & Polyneuropathie, distale Myopathien \\
\hline - asymmetrisch/fokal & $\begin{array}{l}\text { Diabetische Amyotrophie, Motoneuronerkran- } \\
\text { kungen, Entzündliche Nervenerkrankungen wie } \\
\text { die multifokale mororische Neuropathie (MMN) }\end{array}$ \\
\hline \multicolumn{2}{|l|}{ Begleitsymptome } \\
\hline - Myalgien & $\begin{array}{l}\text { Metabolische Myopathien, Proximale myotone } \\
\text { Muskeldystrophie („PROMM“), Myositis }\end{array}$ \\
\hline - Crampi & Metabolische Myopathien, Polyneuropathie \\
\hline - Myotonie & $\begin{array}{l}\text { PROMM, myotone Dystrophie Typ } 1 \text {, nicht } \\
\text { dystrophische Myotonien }\end{array}$ \\
\hline $\begin{array}{l}\text { - initial bereits } \\
\text { deutliche } \\
\text { Muskelatrophien }\end{array}$ & Motoneuronerkrankungen, Polyneuropathie \\
\hline $\begin{array}{l}\text { - Sensibilitätsstörun- } \\
\text { gen }\end{array}$ & Polyneuropathien \\
\hline - Hautveränderungen & Dermatomyositis \\
\hline $\begin{array}{l}\text { - extramuskuläre } \\
\text { Organbeteiligung }\end{array}$ & Entzündliche Myopathien \\
\hline
\end{tabular}

\section{Allgemeine körperliche und klinisch- neurologische Untersuchung}

Die allgemeine körperliche Untersuchung dient der Feststellung einer möglichen extramuskulären Organbeteiligung mit Augenmerk auf Haut, Lunge und Herz sowie der Erhebung möglicher Begleit- und Vorerkrankungen. So finden sich beispielsweise bei der Dermatomyositis typische Hautveränderungen an lichtexponierten Stellen (heliotropes Exanthem), im Bereich der Fingergelenke und am Nagelbett. Eine gefürchtete Komplikation einiger entzündlicher Myopathien ist das Auftreten einer interstitiellen Lungenerkrankung und Fibrose. Zudem kann es im Zuge einiger Myopathien oder im Rahmen neuromuskulärer Übertragungsstörungen zu einer Beteiligung der Atemhilfsmuskulatur kommen. Daher sollte bei entsprechendem Verdacht oder auch als Screening die Bestimmung der Vitalkapazität erfolgen.

Auch eine kardiale Mitbeteiligung ist sowohl bei Myositis, als auch bei verschiedenen erblichen Myopathien bekannt. Die körperliche Untersuchung sollte daher auch ein Augenmerk auf mögliche Zeichen einer kardialen Insuffizienz legen.

Zur Beurteilung der muskulären Schwäche ist eine eingehende neurologische Untersuchung zwingend erforderlich. Dabei sollte die Muskeltrophik (Atrophie, (Pseudo-)Hypertrophie) beurteilt werden und auf das Vorliegen unwillkürlicher Muskelbewegungen wie Faszikulationen geachtet werden.

Die meisten Patienten mit einer neuromuskulären Erkrankung zeigen keine Veränderung des Muskeltonus. Insbesondere erbliche Myopathien sowie Neuropathien können jedoch zu einer Herabsetzung des Muskeltonus führen (Muskelhypotonie). Zudem finden sich häufig abgeschwächte oder gar erloschene Muskeleigenreflexe.

Ein erhöhter Muskeltonus im Sinne einer Spastik weist immer auf eine zentrale Genese der Störung mit Schädigung der Pyramidenbahn hin. Zudem führt eine Schädigung des 1. Motoneurons in der Regel zu gesteigerten Muskeleigenreflexen und Pyramidenbahnzeichen. Zentrale Paresen finden sich bspw. nach einem Schlaganfall, bei Multipler Sklerose (MS), Hirntumoren oder bei der Amyotrophen Lateralsklerose. Letztere stellt in der Diagnostik eine Besonderheit dar, da es neben dem Untergang zentraler Motoneurone auch zu einem Untergang der motorischen Vorderhornzellen kommt. Klinisch finden sich daher neben Zeichen einer zentralen Parese auch schlaffe Paresen, Muskelatrophien und Faszikulationen.

Zur Graduierung der Muskelschwäche bei peripheren und zentralen Paresen erfolgt eine Einschätzung der Kraft anhand der Skala des Medical Research Council (MRC). Die Beurteilung der Kraftgrade erfolgt von 0 bis 5: 0 = keine sichtbare Muskelkontraktion (Plegie); 1 = tastbare Muskelkontraktion; 2 = aktive Bewegung unter Aufhebung der Schwerkraft; 3 = aktive Bewegung gegen die Schwerkraft; 4 = aktive Bewegung gegen leichten Widerstand; $5=$ volle Kraftentfaltung. Es werden alle Muskelgruppen einzeln und jeweils im Seitenvergleich untersucht. Die MRC-Skala kann nicht nur zur initialen Diagnostik verwendet werden, sondern ist auch ein wichtiger Bestandteil der Verlaufsbeurteilung.

Bei Patienten mit einer neuromuskulären Übertragungsstörung wie beispielsweise der Myasthenia gravis lassen sich häufig keine manifesten Paresen in der Untersuchung dokumentieren. Bei klinischem Verdacht oder entsprechender Anamnese einer belastungsabhängigen Muskelschwäche sollte daher eine weitergehende Untersu- 
chung gemäß dem quantitativen Myasthenia-gravis-Score erfolgen [1, 2]. Hierbei werden Ermüdungstest mit Halteversuchen (Kopf, Arme und Beine) und der Simpson-Test über einen definierten Zeitraum durchgeführt.

- Tab. 2 zeigt eine Übersicht der Ursachen von Muskelschwäche und wichtige neuromuskuläre Erkrankungen.

\section{Laboruntersuchungen}

Jede akut aufgetretene, progrediente oder über längere Zeit bestehende muskuläre Schwäche bedarf einer weitergehenden Abklärung. Zunächst sollten orientierende Laboruntersuchungen auch zum Ausschluss anderweitiger Ursachen erfolgen. Neben dem Ausschluss einer Elektrolytstörung, sollten eine Schilddrüsenfunktionsstörung, ein Diabetes mellitus und Cortisolmangel ausgeschlossen werden.

Der Bestimmung der Creatinkinase (CK) als erster Schritt in der Diagnostik von Muskelerkrankungen kommt eine größere Bedeutung bei. Sie ermöglicht einen ersten Überblick über das Ausmaß des Muskelfaseruntergangs. Da es auch nach körperlicher Aktivität zum einem leichten bis moderaten Anstieg der CK kommen kann, sollte die Bestimmung nach Einhaltung körperlicher Schonung erfolgen. Werte über $1000 \mathrm{U} / \mathrm{I}$ (Normwert < $170 \mathrm{U} / \mathrm{I}$ ) lassen an eine muskuläre Erkrankung denken. Auch bei Neuropathien findet sich eine leichte bis mäßige Erhöhung der CK, die jedoch selten mehr als das 10-fache der Norm beträgt [3]. Ein massiver Anstieg der CK auf Werte $>10000 \mathrm{U} / \mathrm{l}$ jedoch weist immer auf eine primär myogene Ursache (Myopathie, Myositis) hin.

Persistierende oder wiederholt erhöht gemessene CK-Werte müssen jedoch auch ohne Vorliegen einer Muskelschwäche an eine neuromuskuläre Erkrankung denken lassen und sollten weiter abgeklärt werden. Darüber hinaus gibt es jedoch auch Myopathien, die keine CK-Erhöhung aufweisen oder bei denen es im Verlauf der Erkrankung zu einer Normalisierung des CK-Wertes kommt.

Bei Verdacht auf das Vorliegen einer metabolischen Myopathie sind weitere spezielle Laboruntersuchungen mit Enzymbestimmung und Bestimmung der Acyl-Carnitin-Spiegel im Serum in einem Stoffwechsellabor notwendig. Erste Hinweise welche Art der Stoffwechselstörung vorliegt, kann der Unterarmbelastungstest liefern. Während ein fehlender Laktatanstieg bei normalem Ammoniakanstieg eher auf eine Glykogenose hindeutet, zeigt sich beim MAD-Mangel ein fehlender Ammoniakanstieg bei normalem Laktatanstieg. Zu den häufigsten metabolischen Myopathien zählen die Glykogenose Typ 2 (Pompe-Erkrankung) bei $\alpha$-GlukosidaseMangel, die Glykogenose Typ 5 (McArdle-Erkrankung) bei Myophosphorylasemangel, der Carnitin-Palmitoyl-Transferase-II-Mangel (CPT-II-Mangel) und der Myoadenolatdesaminase-Mangel (MADMangel).

Mittlerweile ist eine Vielzahl myositis-spezifischer und myositisassoziierter Antikörper identifiziert worden, die bei der Einordnung der entzündlichen Myopathie nützlich sein können. Aufgrund der häufigen Assoziation entzündlicher Myopathien mit anderen Kollagenosen, sollte zudem eine ergänzende rheumatologische Labordiagnostik erfolgen.

Besteht der Verdacht einer neuromuskulären Übertragungsstörung sollten zunächst die am häufigsten vorliegenden Anti-Acetylcholin-Rezeptor-Antikörper (AChR-Ak) bestimmt werden. Bei un- auffälligem Befund kann dann nach weiteren Antikörpern wie Anti-MuSK-Ak (muskelspezifische Tyrosinkinase), Anti-LRP4-Ak (low-density lipoprotein-related-Protein-4), Anti-Agrin-Ak und anti-VGCC-AK (voltage gated calcium channels) gesucht werden. Bei Thymom-assoziierter Myasthenia gravis können Auto-Antikörper gegen AChR, ,Titin, RyR (Ryanodin-Rezeptor), TRPC2, IL12, IFNa und IFNy positiv sein.

Auf die myositis-spezifischen und myositis-assoziierten Antikörper sei im Folgenden nochmal detaillierter eingegangen.

\section{Myositis-spezifische und Myositis-assoziierte Auto-Antikörper}

Im Verlauf der letzten Jahre konnten eine Reihe von Auto-Antikörpern im Serum von Patienten mit entzündlichen Muskelerkrankungen identifiziert werden.

Bislang erfolgt eine Einteilung der Antikörper in Myositis-spezifische und Myositis-assoziierte Antikörper. Letztere treten im Rahmen von systemischen Kollagenosen mit begleitender Entzündung der Skelettmuskulatur auf („Overlap-Myositis“). Die Myositis-spezifischen Auto-Antikörper finden sich mit relativ hoher Spezifität bei einem der übrigen Myositis-Syndrome. Zunehmend werden jedoch auch Überschneidungen beschrieben, so dass eine Differenzierung zwischen „Myositis-spezifisch“ und „Myositis-assoziiert“ in Zukunft aufgehoben werden sollte. Die Antikörper richten sich gegen eine Vielzahl verschiedener nukleärer und zytoplasmatischer Antigene. Häufig persistieren die Antikörper auch unter Therapie. Zudem lässt sich keine sichere Korrelation zwischen Antikörper-Titer und Krankheitsaktivität feststellen. Die Bestimmung der Antikörper liefert somit zusätzliche Informationen in der Einordnung der Myositis-Syndrome, eignet sich jedoch nicht zur Verlaufskontrolle oder zum Therapie-Monitoring.

\section{Apparative neurologische Diagnostik}

\section{Elektrophysiologische Diagnostik}

Die Nadelelektromyografie (EMG) nimmt in der Diagnostik von Myopathien einen großen Stellenwert ein und sollte bei allen Patienten mit dem klinischen Verdacht einer Myopathie durchgeführt werden. Anhand charakteristischer Veränderungen der Muskelaktionspotentiale in der Willküraktivität, lassen sich neurogene und myopathische Schädigungsmechanismen voneinander abgrenzen. Bei Myopathien finden sich niedrigamplitudige, verkürzte und polyphasische Potentiale mit pathologischer Rekrutierung und dichtem Interferenzmuster in der Maximalinnervation. Darüber hinaus ermöglicht die EMG die Detektion myotoner Entladungen. Es empfiehlt sich mehrere Muskeln an unterschiedlichen Extremitäten proximal und distal zu untersuchen.

Zum Ausschluss einer (zusätzlichen) neuropathischen Erkrankung sollten insbesondere bei distalem Verteilungsmuster der Muskelschwäche zudem orientierende sensible und motorische neurographische Untersuchungen erfolgen. Finden sich klinisch Hinweise auf eine neuromuskuläre Übertragungsstörung, kann mittels repetitiver Stimulation ein Dekrement oder Inkrement nachgewiesen werden. 
- Tab. 2 Ursachen von Muskelschwäche und wichtige neuromuskuläre Erkrankungen.

\begin{tabular}{|c|c|c|c|}
\hline & Erkrankung & Symptome & Diagnostik \\
\hline Neuropathien & $\begin{array}{l}\text { z. B. diabetische Polyneuropathie, } \\
\text { autoimmun-entzündliche Neuropathien } \\
\text { wie die chronisch inflammatorische } \\
\text { Polyradikuloneuritis (CIDP) oder die } \\
\text { Multifokale, motorische Neuropathie } \\
\text { (MMN) }\end{array}$ & $\begin{array}{l}\text { Sensibilitätsstörungen (Hyp- und } \\
\text { Dysästhesien, Tiefensensibilitätsstörun- } \\
\text { gen), trophische Störungen, meist distal } \\
\text { symmetrische Verteilung; Sonderformen } \\
\text { mit proximaler oder asymmetrischer/ } \\
\text { fokaler Beteiligung }\end{array}$ & $\begin{array}{l}\text { Umfangreiche Labordiagnos- } \\
\text { tik, Elektrophysiologie (ENG } \\
\text { und EMG) }\end{array}$ \\
\hline \multirow[t]{2}{*}{ Motoneuronerkrankungen } & Amyotrophe Lateralsklerose (ALS) & $\begin{array}{l}\text { Zeichen des } 1 \text {. und } 2 \text {. Motoneurons, } \\
\text { bulbäre Symptome, respiratorische } \\
\text { Insuffizienz }\end{array}$ & $\begin{array}{l}\text { Ausschluss von Differentialdia- } \\
\text { gnosen, klinische Diagnose, } \\
\text { Elektrophysiologie }\end{array}$ \\
\hline & Spinale Muskelatrophien & $\begin{array}{l}\text { Schlaffe, symmetrische Paresen und } \\
\text { Atrophien }\end{array}$ & Molekulargenetik \\
\hline \multicolumn{4}{|l|}{ Myopathien } \\
\hline endokrin & $\begin{array}{l}\text { Hypothyreose,Hypercortisolismus, } \\
\text { Hyperparathyreoidismus }\end{array}$ & & Labordiagnostik \\
\hline Medikamentös/toxisch & $\begin{array}{l}\text { Statinassoziierte Myopathie, Steroidmyo- } \\
\text { pathie, Checkpoint-Inhibitoren, Alkohol }\end{array}$ & & Anamnese \\
\hline \multirow[t]{4}{*}{$\begin{array}{l}\text { erblich mit Beginn im } \\
\text { Erwachsenenalter }\end{array}$} & FSHD Typ 1 und 2 & $\begin{array}{l}\text { Fazies myopathica, Atrophie und Schwäche } \\
\text { der Schultergürtelmuskulatur, Scapula alata }\end{array}$ & \multirow[t]{4}{*}{ Molekulargenetik } \\
\hline & Myotone Dystrophie Typ II (PROMM) & $\begin{array}{l}\text { proximale Schwäche, Myotonie, wenig } \\
\text { Atrophien, kardiale Reizleitungsstörung }\end{array}$ & \\
\hline & Gliedergürteldystrophie & Beginn meist im Beckenbereich & \\
\hline & Myofibrilläre Myopathie & Klinisch heterogen & \\
\hline \multirow[t]{4}{*}{ metabolisch } & MAD-Mangel & $\begin{array}{l}\text { Belastungsabhängige Myalgien, Crampi, } \\
\text { vorzeitige Ermüdbarkeit der Muskulatur }\end{array}$ & $\begin{array}{l}\text { Biochemischer oder histoche- } \\
\text { mischer Nachweis von } \\
\text { MAD-Mangel }\end{array}$ \\
\hline & Glykogenose Typ II (Pompe-Erkrankung) & $\begin{array}{l}\text { proximale Schwäche, Beteiligung der } \\
\text { Atemhilfsmukulatur, }\end{array}$ & $\begin{array}{l}\text { Nachweis } \alpha \text {-Glukosidase- } \\
\text { Mangel, Molekulargenetik, } \\
\text { Muskelbiopie }\end{array}$ \\
\hline & Glykogenose Typ V (McArdle-Krankheit) & $\begin{array}{l}\text { Belastungsabhängige Crampi/Kontrakturen } \\
\text { und Schwäche, im Verlauf persitierend, } \\
\text { „second-wind“-Phänomen, Gefahr der } \\
\text { Rhabdomyolyse }\end{array}$ & $\begin{array}{l}\text { Nachweis Muskelphosphoryla- } \\
\text { xe-Mangel in der Muskelbiop- } \\
\text { sie, Molekulargenetik }\end{array}$ \\
\hline & CPT-II-Mangel & Episodische Rhabdomyolyse, Myoglobinurie & \multirow[t]{4}{*}{ Molekulargenetik } \\
\hline \multirow[t]{4}{*}{ entzündlich } & Dermatomyositis (DM) & $\begin{array}{l}\text { Proximale, symmetrische Muskelschwäche, } \\
\text { Atrophien, Myalgien; charakteristische } \\
\text { Hautveränderungen }\end{array}$ & \\
\hline & Polymyositis (PM) & $\begin{array}{l}\text { Proximale, symmetrische Muskelschwäche, } \\
\text { Atrophien, Myalgien }\end{array}$ & \\
\hline & Nekrotisierende Myopathie (NM) & $\begin{array}{l}\text { Proximale, symmetrische Muskelschwäche, } \\
\text { Atrophien, Myalgien }\end{array}$ & \\
\hline & Einschlusskörpermyositis (IBM) & $\begin{array}{l}\text { Asymmetrische Verteilung der Paresen, v.a. } \\
\text { Fingerbeuger und Kniestrecker, dtl. } \\
\text { Atrophien, Dysphagie }\end{array}$ & $\begin{array}{l}\text { cN1A-Antikörper, Muskelbiop- } \\
\text { sie inkl. Elektronenmikroskopie }\end{array}$ \\
\hline \multirow[t]{2}{*}{$\begin{array}{l}\text { Neuromuskuläre Übertra- } \\
\text { gungsstörung }\end{array}$} & Myasthenia gravis (MG) & $\begin{array}{l}\text { Belastungsabhängige Ermüdbarkeit, Ptosis, } \\
\text { Doppelbilder, Schluckstörung, Dysarthrie }\end{array}$ & $\begin{array}{l}\text { Acetylcholin-Rezeptor-Antikör- } \\
\text { per, MuSK-Ak, LRP4-Ak, } \\
\text { Agrin-Ak; Dekrement in der } \\
\text { repetitiven Stimulation }\end{array}$ \\
\hline & $\begin{array}{l}\text { Lambert-Eaton-Myasthenie-Syndrom } \\
\text { (LEMS) }\end{array}$ & $\begin{array}{l}\text { proximal betonte, belastungsabhängige } \\
\text { Schwäche, Augenmuskelbeteiligung; } \\
\text { autonome Störung; paraneoplastische } \\
\text { Genese möglich }\end{array}$ & $\begin{array}{l}\text { VGCC-Antikörper; Inkrement in } \\
\text { der repetitiven Stimulation }\end{array}$ \\
\hline
\end{tabular}




\section{Bildgebende Diagnostik}

Seit einigen Jahren nehmen in der Diagnostik von Myopathien auch bildgebende Verfahren einen größeren Stellenwert ein.

Die Myosonographie ist eine leicht verfügbare, nicht invasive Untersuchung in der Diagnostik neuromuskulärer Erkrankungen. Neben Muskelatrophien und -hypertrophien können auch Veränderungen in der Echogenität des Muskels und somit indirekt Umbauvorgänge dargestellt werden. Zudem gewinnt die Myosonographie zunehmend auch in der Detektion von Faszikulationen in der Diagnostik der Amyotrophen Lateralsklerose an Bedeutung.

Als wichtigstes bildgebendes Verfahren bei Myopathien hat sich die Muskel-MRT durchgesetzt. Sie erlaubt eine gute Darstellung des Verteilungsmusters der beteiligten Muskeln. Ein fettiger Umbau der Muskulatur lässt sich in den T1-gewichteten Sequenzen darstellen. Entzündliche Veränderungen lassen sich zum einem mittels kontrastmittelgestützter Sequenzen, zum anderen indirekt durch Darstellung des begleitenden Ödems dokumentieren. Insbesondere mithilfe der T2- und fettunterdrückten STIR-Sequenz (short tau inversion recovery) lassen sich hyperintense Areale im Bereich des Ödems mit hoher Sensitivität darstellen. Die MuskelMRT hilft somit auch bei der Auswahl geeigneter Stellen für die Muskelbiopsie. Zudem konnten mehrere Untersuchungen eine Korrelation von Krankheitsaktivität und Signalveränderungen in der T1, T2 und STIR dokumentieren [4, 5]. Somit kann die Untersuchung auch zur Verlaufsbeurteilung nützlich sein.

\section{Muskelbiopsie}

Die Muskelbiopsie ist weiterhin von essentieller Bedeutung in der Diagnostik von Myopathien. Neben der histologischen Begutachtung erlauben immunhistochemische, molekularbiologische, biochemische und ggf. elektronenmikroskopische Untersuchungen eine weitere Einordnung und Differenzierung verschiedener Myopathien. Sie erfolgt über einen offenen Hautschnitt in ausreichender Größe unter Lokalanästhesie.

Die Untersuchung sollte nach Möglichkeit in einem zertifizierten, neuromuskulären Zentrum erfolgen. Zudem empfiehlt sich die Untersuchung vor Einleitung einer immunsuppressiven Therapie vorzunehmen, um falsch negative Ergebnisse zu vermeiden. Andernfalls muss die bereits begonnene immunsuppressive Therapie nach Möglichkeit vor Durchführung der Muskelbiopsie über längere Zeit pausiert werden. Für eine größtmögliche diagnostische Aussagekraft ist zudem der Auswahl des Biopsieortes von großer Bedeutung. Grundsätzlich sollte ein klinisch betroffener Muskel gewählt werden, der nach Möglichkeit eine mittelgradige Parese aufweist. Die Muskel-MRT kann helfen, eine geeignete Biopsiestelle bspw. über Darstellung der entzündlich veränderten Areale auszuwählen. Liegt bereits ein ausgeprägter fettiger oder bindegewebiger Umbau der Muskulatur vor, ist die Aussagekraft einer Muskelbiopsie sehr eingeschränkt. Zudem gilt es zu beachten, dass die Muskelbiopsie nicht aus einem Muskel erfolgen sollte, welcher innerhalb der letzten 2 Wochen mittels Nadel-EMG untersucht wurde.

\section{Erweiterte Diagnostik}

Die erweiterte Diagnostik orientiert sich an der zugrundeliegenden neuromuskulären Erkrankung.
Bei Vorliegen einer Myasthenia gravis sollte zum Ausschluss eines Thymoms eine bildgebende Diagnostik des Thorax bzw. Mediastinums erfolgen (CT/MRT). Eine Reihe von entzündlichen Myopathien und das Lambert-Eaton-Myasthenie-Syndrom (LEMS) können mit dem Vorliegen einer Tumorerkrankung assoziiert sein. In diesem Falle sollte daher eine eingehende Tumorsuche erfolgen.

Zudem finden sich bei entzündlichen Myopathien häufig eine Überlappung mit anderen Kollagenosen sowie extramuskuläre Organbeteiligungen. Auch andere insbesondere erbliche Myopathien können bspw. eine kardiale Mitbeteiligung im Sinne einer Kardiomyopathie oder Reizleitungsstörung bedingen. Unter Berücksichtigung des jeweiligen klinischen Syndroms, sollte daher eine sorgfältige weiterführende Diagnostik erfolgen. Dies beinhaltet eine Lungenfunktionsprüfung, Bildgebung der Lunge, EKG, Echokardiografie, opthalmologische, endokrinologische oder weitere rheumatologische Diagnostik.

Da ein großer Teil von Patienten mit neuromuskulären Erkrankungen eine teils klinisch inapparente Dysphagie aufweist, sollten eine logopädische Mitbeurteilung und eine flexible Endoskopie (FEES) oder Videofluoroskopie erfolgen.

Bei Verdacht auf eine erbliche Myopathie erfolgt die Überweisung an ein humangenetisches Zentrum zur Einleitung einer molekulargenetischen Diagnostik.

\section{Neuromuskuläre Erkrankungen mit Leitsymptom Muskelschwäche}

\section{Neuromuskuläre Übertragungsstörungen}

Zu den neuromuskulären Übertragungsstörungen gehören unter anderem die Myasthenia gravis sowie das Lambert-Eaton-Myasthenie-Syndrom. Die Erkrankungen führen zu einer belastungsabhängigen Schwäche der quergestreiften Muskulatur.

Bei der Myasthenia gravis (MG) kommt es zu Beginn häufig zu einem isolierten Befall der Augenmuskeln mit Auftreten einer Ptosis und von Doppelbildern (okuläre Myasthenie). Beim Großteil der Patienten (80-90\%) kommt es innerhalb von 2 Jahren zu einer Generalisierung der Erkrankung mit Ausdehnung auf die Skelett-, Schlund- und Atemhilfsmuskulatur [6]. Dabei werden leichte, mittelschwere und schwere generalisierte Myasthenie entsprechend des klinischen Schweregrades unterschieden. Die MG ist eine Antikörper-vermittelte Erkrankung, bei der es durch Blockade der postsynaptischen Acetylcholin-Rezeptoren zu einer Störung der neuromuskulären Erregungsübertragung kommt. Insbesondere bei jungen Patienten findet sich nicht selten der Nachweis eines Thymusrestes oder Thymoms. In mehr als 85 \% der Fälle lassen sich Acetylcholin-Rezeptor-Antikörper im Serum von Patienten detektieren. Weitere Antikörper sind gegen MuSK, LRP4, Agrin oder Titin gerichtet. Über die Komplementaktivierung führen die Antikörper zudem zu einer Zerstörung der muskulären Endplatte [7]. Eine Reihe von Medikamenten können zu einer Exazerbation der Myasthenie führen und müssen gemieden werden. Eine gefürchtete Komplikation der Erkrankung ist die myasthene Krise, die einer intensivmedizinischen Behandlung bedarf.

Die Behandlung der MG erfolgt zum einen symptomatisch über die Einnahme von Acetylcholinesterase-Hemmern wie Pyridostigmin, zum anderen immunsuppressiv. Mittel der 1. Wahl sind nach 
- Tab. 3 Überblick Myositis-spezifische (MSA) und Myositis-assoziierte Auto-Antikörper (MAA).

\begin{tabular}{|c|c|c|}
\hline & Assoziierte Syndrome/Phänotyp & $\begin{array}{l}\text { Häufig- } \\
\text { keit } \\
\text { (\%) }\end{array}$ \\
\hline \multicolumn{3}{|c|}{ MSA } \\
\hline Anti-Synthetase-Ak & \multirow[t]{9}{*}{ Anti-Synthetase-Syndrom } & \\
\hline Anti-jo1 & & $10-25$ \\
\hline Anti-OJ & & \\
\hline Anti-EJ & & \\
\hline Anti-PL12 & & \\
\hline Anti-PL7 & & \\
\hline Anti-Ha & & \\
\hline Anti-Zo & & \\
\hline \multicolumn{2}{|l|}{ Anti-KS } & \\
\hline Anti-Mi2 & Klassische DM & $5-10$ \\
\hline Anti-TIF-1Y-Ak & $\begin{array}{l}\text { DM, Assoziation mit Malignomen, } \\
\text { schwerer Verlauf bei juveniler DM }\end{array}$ & $\sim 20$ \\
\hline MDA5-Ak & $\begin{array}{l}\text { Amyopathische DM, rasch } \\
\text { progrediente Lungenbeteiligung }\end{array}$ & $10-50$ \\
\hline Anti-SRP & $\begin{array}{l}\text { Statin-assoziierte nekrotisierende } \\
\text { Myositis (NM) }\end{array}$ & 5 \\
\hline Anti-p140 & $\begin{array}{l}\text { interstitielle Lungenerkrankung, } \\
\text { juvenile DM mit Kalzinose }\end{array}$ & 5 \\
\hline Anti-HMGCR & NM & 6 \\
\hline Mup44/cN1A-Ak & Einschlusskörpermyositis (IBM) & $\sim 50$ \\
\hline \multicolumn{3}{|c|}{ MAA } \\
\hline Anti-SSA/Ro & $\begin{array}{l}\text { Höheres Risiko für schwere } \\
\text { Lungenbeteiligung bei zusätzlichen } \\
\text { Jo1-Antikörpern }\end{array}$ & bis 30 \\
\hline Anti-SSB/La & Sjögren-Syndrom & bis 14 \\
\hline Anti-U1RNP & $\begin{array}{l}\text { Mischkollagenosen, systemischer } \\
\text { Lupus erythematodes, Skleroder- } \\
\text { mie }\end{array}$ & 10 \\
\hline Anti-U2RNP & $\begin{array}{l}\text { Sjögren-Syndrom, Overlap von } \\
\text { Myositis und Sklerodermie }\end{array}$ & 15 \\
\hline Anti-PmSCl & $\begin{array}{l}\text { Overlap von Myositis und } \\
\text { Sklerodermie }\end{array}$ & 10 \\
\hline Anti-Ku & $\begin{array}{l}\text { Overlap von Myositis und } \\
\text { Sklerodermie }\end{array}$ & $20-30$ \\
\hline
\end{tabular}

(Amlani et al. 2019; Betteridge und McHugh 2015; Satoh et al. 2017, Fitzner und Schmidt 2016).

wie vor der Einsatz von Glukokortikosteroiden und Azathioprin. Schwere Verläufe können den Einsatz von Rituximab oder Eculizumab notwendig machen [8]. In der Behandlung der myasthenen Krise kommen Immunglobuline und Plasmaaustauschverfahren zum Einsatz.

Das LEMS kann als paraneoplastisches oder autoimmunologisches Syndrom auftreten. Durch Blockade der präsynaptischen spannungsabhängigen Calciumkanäle kommt es wie bei der MG zu einer Störung der neuromuskulären Übertragung. Beim paraneoplastischen LEMS finden sich bei zwei Drittel der Patienten Anti-SOX1-Antikörper. Klinisch finden sich eine proximal betonte belastungsabhängige Schwäche, eine Hyporeflexie und autonome Störungen. Eine sorgfältige Tumorsuche sollte nicht nur mit Diagnosestellung, sondern auch im Verlauf der Erkrankung regelmäßig erfolgen [9].

\section{Inflammatorische Myopathien (Myositiden)}

Bei den Myositiden handelt es sich um eine Gruppe erworbener, entzündlicher Myopathien, die zu einer Muskelschwäche und Muskelatrophie führen. Es werden 5 verschiedene Subtypen anhand charakteristischer klinischer und pathologischer Kriterien unterschieden: die Polymyositis (PM), Dermatomyositis (DM), nekrotisierende Myopathie (NM), Myositis bei Overlap-Syndrom und Einschlusskörpermyositis (inclusion body myositis, IBM).

Bis auf die Einschlusskörpermyositis kommt es in der Regel zu einem akuten bis subakuten Beginn der Erkrankung. Neben einer proximal betonten Muskelschwäche und Muskelatrophie, berichten die Patienten nicht selten auch über Myalgien. Bei der Dermatomyositis finden sich zudem klassische Hausveränderungen. Darüber hinaus weisen die DM, PM, NM und Overlap-Myositis häufig extramuskuläre Organmanifestationen und Assoziation mit Tumorerkrankungen auf. Das Anti-Synthetase-Syndrom beschreibt einen Symptomkomplex aus Myositis, interstitieller Lungenerkrankung, Arthritis und Raynau-Phänomen. Der häufigste und am besten charakterisierte Anti-Synthetase-Antikörper ist Jo-1. Es ist noch nicht abschließend geklärt, ob die Myositis bei Antisynthetase-Syndrom als Teil der Overlap-Myositis oder als eigenständige Erkrankung zu charakterisieren ist. Pathophysiologisch wird für die PM, DM, NM und Myositis bei Overlap-Syndromen eine autoimmunologische Genese angenommen. Die Therapie basiert auf Einleitung einer immunsuppressiven Therapie. Zum Einsatz kommen Glukokortikosteroide, kortikoidsparende Immunsuppressiva wie Azathioprin, Mycophenolat-Mofetil oder MTX und intravenöse Immunglobuline. Bei schweren Verläufen finden sich positive Daten für den Einsatz von Rituximab und Cyclophosphamid [10,11]. Dank intensiver Forschung auf dem Gebiet der Myositis in den letzten 10 Jahren, stehen heute einige biologische Präparate und JAK-Inhibitoren „in der Pipeline“. Eine Ausnahme stellt die Einschlusskörpermyositis dar, die in der Muskelbiopsie entzündliche und degenerative Veränderungen aufweist und bei der immunsuppressive Therapien unwirksam scheinen.

In letzter Zeit wurden auch zunehmend therapie-assoziierte entzündliche Myopathien beschrieben. So kann bspw. die Einnahme von Statinen zu einer nekrotisierenden Myopathie führen. Eine mögliche statin-induzierte Myotoxizität ist schon lange bekannt und kann von Myalgien oder einer asymptomatischen CK-Erhöhung bis hin zur Rhabdomyolyse reichen. In der Regel sistieren die Beschwerden mit Wechsel oder Absetzen der Medikation. 2007 beschrieben Needham et al. erstmals eine fortschreitende Myopathie auch nach Beendigung der Statintherapie [12]. Histologisch zeigte sich das Bild einer nekrotisierenden inflammatorischen Myopathie mit erhöhter MHC-I-Expression, die eine autoimmune Genese nahelegte. Die Hypothese einer autoimmunologischen Pathogenese wird zusätzlich untermauert durch die Identifikation von Antikörpern gegen die 3-Hydroxy-3-methylglutaryl-Coenzym A Redukta- 
se (HMGCoAR-Ak) und das Ansprechen auf immunsuppressive Therapien [13] $>$ Tab. 3.

Auch der Einsatz moderner Krebstherapien mit Checkpoint-Inhibitoren kann nachweislich zu autoimmunen Nebenwirkungen an verschiedenen Organsystemen führen [14]. Neben Myositiden wurden bei Patienten auch Fälle von Enzephalopathien, Myasthenia gravis und Guillain-Barré-Syndrom (GBS) beschrieben [15, 16].

\section{Erbliche Myopathien mit Beginn im Erwachsenenalter}

Eine Vielzahl von Myopathien können sich erst im Erwachsenenalter manifesterieren. Hierzu zählen beispielsweise die Myotone Dystrophie Typ II (proximale myotone Myopathie, PROMM), die Fazioskapulohumerale Muskeldystrophie (FSHD) sowie einige Unterformen der Gliedergürteldystrophien und myofibrillären Myopathien.

Die Myotone Dystrophie Typ II weist einen autosomal-dominanten Erbgang auf und manifestiert sich im frühen bis mittleren $\mathrm{Er}$ wachsenenalter. Klinisch finden sich eine proximale Schwäche der Extremitätenmuskulatur ohne deutliche Muskelatrophien bei Hypertrophie der Wadenmuskulatur, muskelkaterartige Schmerzen und Zeichen der Myotonie. Diese stehen im Gegensatz zur myotonen Dystrophie Typ I jedoch nicht im Vordergrund. Es kann zu kardialen Reizleitungsstörungen und endokrinologischen Veränderungen mit Schilddrüsenfunktionsstörung, Diabetes mellitus und Hodenatrophie sowie Entwicklung eines Kataraktes kommen.

Diagnostisch finden sich eine erhöhte CK und erhöhte Leberwerte sowie der Nachweis von myotonen Salven im EMG. Die Diagnose erfolgt molekulargenetisch über den Nachweis einer CCTGExpansion im Gen CNBP (ZNF9) auf Chromosom 3q21.3.

Die FSHD weist wie der Name bereits vermuten lässt einen Befall der Gesichts- und Schultergürtelmuskulatur auf. Darüber hinaus finden sich Muskelschmerzen, eine Degeneration der Retina und eine Innenohrschwerhörigkeit. Die Erkrankung wird autosomal-dominant vererbt, in bis zu einem Drittel der Fälle liegen jedoch auch Neumutationen vor.

Darüber hinaus zeigen die hereditären metabolischen Myopathien wie der primäre Myadenylat-Desaminase-Mangel (MAD-Mangel), die Glykogenosen Typ II (Pompe-Erkrankung) und V (McArdle-Erkrankung) und CPT-II-Mangel, einen Beginn im Erwachsenenalter.

Der MAD-Mangel ist die häufigste metabolische Myopathie und kann auch sekundär bei Vorliegen anderer Myopathien auftreten. Er zeichnet sich klinisch durch Muskelschmerzen und Crampi sowie eine belastungsabhängige vorzeitige Ermüdbarkeit der Muskulatur aus. Die Diagnose erfolgt über den histochemischen oder biochemischen Nachweis eines MAD-Mangels. Die häufigste Glykogenose ist die autosomal-rezessiv vererbte Pompe-Erkrankung, bei der es aufgrund des $\alpha$-Glukosidase-Mangels zu einem gestörten Glykogenabbau kommt. Bei Erkrankungsbeginn im Erwachsenenalter finden sich eine proximale Muskelschwäche und -atrophie mit Beteiligung der Atemhilfsmukulatur. Diagnostisch erfolgt der Nachweis einer verminderten Aktivität der sauren Maltase auf Trockenblut bzw. der humangenetische Nachweis verschiedener Mutationen auf Chromosom 17q23. Therapeutisch erfolgt neben der symptomatischen Therapie der Enzymersatz über rekombinante humane $\alpha$-1,4-Glukosidase (Myozyme).

\section{Neuropathien}

Die häufigsten Ursachen für Polyneuropathien sind der Diabetes mellitus und schädlicher Alkoholgebrauch. Darüber hinaus gibt es jedoch eine Vielzahl weiterer metabolischer, nutritiver, infektiologischer, medikamentös-toxischer und immunvermittelter Ursachen erworbener Polyneuropathien.

Motorische Symptome bei Polyneuropathien finden sich überwiegend distal betont im Bereich der Fußheber sowie im Bereich der kleinen Hand- und Fußmuskeln. Ausnahmen stellen die chronisch-inflammatorisch demyelinisierenden Polyneuropathie (CIDP) und ihre Sonderform die multifokale motorische Neuropathie (MMN) dar. Bei der CIDP finden sich auch proximal gelegene Paresen, während die MMN häufig einen asymmetrischen Befall mit ausgeprägten Muskelatrophien aufweist. Weitere PolyneuropathieSyndrome mit überwiegend motorischem Befall sind die diabetische Amyotrophie, hereditäre Neuropathien, das Guillain-BarréSyndrom sowie die Porphyrie.

\section{Motoneuronerkrankungen}

Die häufigste Form der Motoneuronerkrankungen ist die Amyotrophe Lateralsklerose (ALS). Mittlerweile wird die ALS als Multisystemerkrankung verstanden, bei der es zu einer Schädigung der motorischen Nervenzellen im Bereich des motorischen Kortex (1. Motoneuron), der bulbären motorischen Hirnnervenkerne sowie der motorischen Vorderhornzellen im Spinalmark kommt. Klinisch finden sich daher Schädigungszeichen des 1. und 2. Motoneurons mit Auftreten zentraler und periphere Paresen. Patienten entwickeln progrediente, zu Beginn oftmals asymmetrische Paresen und Muskelatrophien mit Beteiligung der Atemhilfsmuskulatur sowie eine bulbäre Symptomatik mit Dysarthrie und Dysphagie. Darüber hinaus können durchaus sensible Symptome und neuropsychologische Defizite vorliegen. Die Diagnostik erfolgt klinisch anhand definierter Diagnosekriterien (revidierte El Escorial-Kriterien) und nach Ausschluss von Differentialdiagnosen [17]. Zu Beginn der Erkrankung sind Zeichen des 1. Motoneurons wie Spastik und Pyramidenbahnzeichen häufig maskiert und können die diagnostische Sicherheit einschränken. Elektrophysiologische Untersuchungen und die Bestimmung der Neurofilamente im Serum und Liquor können die Diagnose stützen. Es existiert weiterhin keine kausale Therapie, so dass die symptomatische und supportive Therapie im Vordergrund stehen.

\section{Zusammenfassung und Fazit für die Praxis}

Eine muskuläre Schwäche weist nicht selten auf das Vorliegen einer neuromuskulären Erkrankung hin. Daher sollte bei jeder akut aufgetretenen, wiederkehrenden oder progredienten muskulären Schwäche eine eingehende differentialdiagnostische Abklärung erfolgen. Da es sich bei der Mehrzahl neuromuskulärer Erkrankungen um seltene Krankheitsbilder handelt, sollte bei begründetem Verdacht eine Zuweisung zu einem Neurologen oder neuromuskulären Zentrum erfolgen. Diagnostische Schritte beinhalten neben laborchemischen Untersuchungen, elektrophysiologische, bildgebende und histologische Untersuchungen. Die Behandlung der entzündlichen Myopathien sollte entsprechend den interdisziplinären Leitlinien in enger Zusammenarbeit zwischen Rheumatologen, Dermatologen und Neurologen stattfinden. 


\section{Danksagung}

Die Autoren sind Mitglieder des Europäischen Referenznertzwerks für seltene Neuromuskuläre Erkrankungen (ERN EURO-NMD).

\section{Interessenkonflikt}

M.F. hat keine Interessenkonflikte. J.S. hat Beraterhonorare, Reisekostenerstattungen oder Projektförderungen erhalten von Alnylam, Argenx, Bayer, Biogen, BioMarin, Biotest, CSL Behring, Euroimmun, Grifols, Kezar, LFB, Novartis, Octapharma, Pfizer, UCB.

\section{Literatur}

[1] Barohn R], Mclntire D, Herbelin L et al. Reliability testing of the quantitative myasthenia gravis score. Ann N Y Acad Sci 1998; 841: 769-772. doi:10.1111/j.1749-6632.1998.tb11015.x

[2] Tindall RS, Rollins JA, Phillips JT et al. Preliminary results of a double-blind, randomized, placebo-controlled trial of cyclosporine in myasthenia gravis. N Engl J Med 1987; 316: 719-724. doi:10.1056/ NEJM198703193161205

[3] Chahin N, Sorenson EJ. Serum creatine kinase levels in spinobulbar muscular atrophy and amyotrophic lateral sclerosis. Muscle Nerve 2009; 40: 126-129. doi:10.1002/mus.21310

[4] Milisenda JC, Collado MV, Pinal-Fernandez I et al. Correlation between quantitative and semiquantitative magnetic resonance imaging and histopathology findings in dermatomyositis. Clin Exp Rheumatol 2019; 37: 633-640

[5] Tomasová Studynková J, Charvát F, Jarosová K et al. The role of MRI in the assessment of polymyositis and dermatomyositis. Rheumatology (Oxford) 2007; 46: 1174-1179. doi:10.1093/rheumatology/kem088

[6] Robertson NP, Deans ], Compston DA. Myasthenia gravis: a population based epidemiological study in Cambridgeshire, England. J Neurol Neurosurg Psychiatry 1998; 65: 492-496. doi:10.1136/jnnp.65.4.492

[7] Masuda T, Motomura M, Utsugisawa $\mathrm{K}$ et al. Antibodies against the main immunogenic region of the acetylcholine receptor correlate with disease severity in myasthenia gravis. J Neurol Neurosurg Psychiatry 2012; 83: 935-940. doi:10.1136/jnnp-2012-302705
[8] Howard JF, Utsugisawa K, Benatar M et al. Safety and efficacy of eculizumab in anti-acetylcholine receptor antibody-positive refractory generalised myasthenia gravis (REGAIN): a phase 3, randomised, double-blind, placebo-controlled, multicentre study. Lancet Neurol 2017; 16: 976-986. doi:10.1016/S1474-4422(17)30369-1

[9] Titulaer M], Lang B, Verschuuren J]. Lambert-Eaton myasthenic syndrome: from clinical characteristics to therapeutic strategies. Lancet Neurol 2011; 10: 1098-1107. doi:10.1016/S14744422(11)70245-9

[10] Oddis CV, Reed AM, Aggarwal R et al. Rituximab in the treatment of refractory adult and juvenile dermatomyositis and adult polymyositis: a randomized, placebo-phase trial. Arthritis Rheum 2013; 65: 314-324. doi:10.1002/art.37754

[11] Fasano S, Gordon P, Hajji R et al. Rituximab in the treatment of inflammatory myopathies: a review. Rheumatology (Oxford) 2017; 56: 26-36. doi:10.1093/rheumatology/kew146

[12] Needham M, Fabian V, Knezevic W et al. Progressive myopathy with up-regulation of MHC-I associated with statin therapy. Neuromuscul Disord 2007; 17: 194-200. doi:10.1016/j.nmd.2006.10.007

[13] Mammen AL, Chung T, Christopher-Stine L et al. Autoantibodies against 3-hydroxy-3-methylglutaryl-coenzyme $A$ reductase in patients with statin-associated autoimmune myopathy. Arthritis Rheum 2011; 63: 713-721. doi:10.1002/art.30156

[14] Postow MA, Sidlow R, Hellmann MD. Immune-Related Adverse Events Associated with Immune Checkpoint Blockade. N Engl J Med 2018; 378: 158-168. doi:10.1056/NEJMra1703481

[15] Larkin J, Chmielowski B, Lao CD et al. Neurologic Serious Adverse Events Associated with Nivolumab Plus Ipilimumab or Nivolumab Alone in Advanced Melanoma, Including a Case Series of Encephalitis. Oncologist 2017; 22: 709-718. doi:10.1634/theoncologist.2016-0487

[16] Cuzzubbo S, Javeri F, Tissier M et al. Neurological adverse events associated with immune checkpoint inhibitors: Review of the literature. Eur J Cancer 2017; 73: 1-8. doi:10.1016/j.ejca.2016.12.001

[17] Brooks BR, Miller RG, Swash M et al. El Escorial revisited: revised criteria for the diagnosis of amyotrophic lateral sclerosis. Amyotroph Lateral Scler Other Motor Neuron Disord 2000; 1: 293-299. doi:10.1080/146608200300079536 